\section{俩 Heighten Science \\ P U B L I C A T I O N S Corporation \\ ISSN \\ 2576-9529}

\title{
Can bloodstream infection be predicted by nasal culture in hemodialysis patients?
}

\author{
Behrooz Broumand ${ }^{1 *}$, Shahram Boroumandi ${ }^{1,2}$, Zahra Rahbar ${ }^{1}$, \\ Mehrdad Sheikhvatan ${ }^{3}$ and Maryam Shahroukh ${ }^{1}$ \\ 'Pars Advanced and Minimally Invasive Medical Manners Research Center, Pars Hospital, Iran \\ University of Medical Sciences, Tehran, Iran \\ 2Islamic Azad University, Tehran Medical Sciences Branch, Tehran, Iran \\ ${ }^{3}$ Department of Neurology, Heidelberg University Hospital, Heidelberg, Germany
}

*Address for Correspondence: Dr. Behrooz Broumand, Pars Advanced and Minimally Invasive Medical Manners Research Center, Pars Hospital, Iran University of Medical Sciences, Tehran, Iran, Tel: +982188952035; Fax: +982188989690; Email: v4broumand@yahoo.com

Submitted: 22 October 2018

Approved: 12 April 2019

Published: 15 April 2019

Copyright: ๑ 2019 Broumand B, et al. This is an open access article distributed under the Creative Commons Attribution License, which permits unrestricted use, distribution, and reproduction in any medium, provided the original work is properly cited

Keywords: Infection; Renal dialysis; Nasal lavage fluid

Check for updates

\section{Summary}

The blood and drainage cultures are suggested for early diagnosis of bloodstream infection (BSI), which are time consuming and laborious. Nasal colonization of bacteria is one of the modalities, occasionally can predict BSI. We hypothesized that nasal culture, as an accessible fluid may be helpful to predict future BSI in hemodialysis patients. The present prospective study evaluated 63 patients undergoing maintenance hemodialysis at the Pars hospital dialysis center, Tehran, Iran, from November 2015 until February 2016. Nasal fluid of patients were collected from the $1-\mathrm{cm}$ internal anterior part of both nostrils of patients by a sterile swab and cultured in Trypticase soy agar. All patients were followed for three months for BSI. The results of first nasal fluid sample revealed that $33.3 \%$ in first sampling and $27.0 \%$ in sampling had positive nasal fluid culture. The type of bacteria in all positive cases was Staphylococcus aureus. The rate of BSI infection in the patients with positive and negative first nasal fluid culture was $9.5 \%$ and $2.4 \%$ respectively with no significant difference. We found also no significant association between BSI positivity and nasal culture results so that positive BSI was revealed in $5.9 \%$ of patients with positive nasal fluid culture and $4.3 \%$ in those with negative nasal fluid culture with no meaningful difference. None of the baseline variables including age and gender, underlying risk factor, access, or duration of dialysis was associated with BSI positivity. In hemodialysis patients, BSI may not be predicted by nasal fluid culture positivity.

\section{Introduction}

End stage renal disease (ESRD) unless in renal transplanted patients is associated with lifelong dependence of patients on maintenance dialysis, which can affect patients' quality of life [1] and is associated with greater risk of morbidity and mortality [2]. One of the most important complications is bloodstream infection (BSI), which is the most common cause of hospitalization and the second most common cause of mortality in hemodialysis patients [3]. The overall incidence of BSI among those patients receiving maintenance hemodialysis due to ESRD remains high [4]. Infection acquisition in hemodialysis facilities is mainly due to non-compliance with health principles and in this regard, all guidelines have emphasized following the principles of infection control for preventing BSI in such vulnerable population [5]. At first attempt, medical directors and clinicians should aware the infection control practice guidelines particularly the ways to colonize and then spread the infection. First, it should be noted that about $70 \%$ of BSIs are related to vascular access especially central venous catheters [6,7]. These accesses are responsible for spreading a variety of bacterial and even viral infections [8].

In addition to the access-related infections, infection-related hospitalization of 
hemodialysis patients is associated with low albumin concentrations, immobility, and residence in a day care center [9]. In addition, Staphylococcus aureus in blood culture, and antibiotic resistance are important factors associated with greater risk of mortality and morbidity of BSI in hemodialysis patients [10]. Moreover, persistent nasal colonization with resistant $S$. aureus is suggested as an important risk factor associated with increased all-cause mortality rate [11]. Thus, nasal colonization by $S$. aureus in hemodialysis patients is considered as a risk factor associated with bacteremia and general infection, especially for infections of endogenous origin [12] and its eradication may decrease the infection rate [13]. Moreover, coagulase-negative staphylococci and diphteroids may be positive in one-fourth of patients' nasal vestibules [14]. Therefore, routine nasal fluid culture can be considered an easy access route to predict and prevent future infections. In this regard, some earlier studies could find the evidences on this claim. As shown by Pujol et al. [15], nasal carriers of S. aureus are at increased risk for bacteremia induced by S. aureus as compared to non-carriers. Another evidence has been also published by Chow et al on utilizing topical and oral therapy for eradication of staphylococcal nasal leading decrease in bloodstream infections [16]. Therefore, we hypothesized that nasal fluid culture, as an accessible fluid may be able to predict which maintence hemodialysis patients are prone to BSI.

\section{Methods}

\section{Study design}

This prospective study evaluated hemodialysis patients regarding the association between nasal colonization and BSI. The protocol of the study was approved by Ethics committee of Iran University of Medical Sciences. The study protocol and objectives were first explained to all participants and patients who were willing to participate in the study were recruited after signing the written informed consent. All study steps were designed and implemented based on principles of Helsinki's Declaration.

Patients undergoing chronic hemodialysis at dialysis center of Pars hospital, Tehran, Iran, from November 2015 until February 2016, and were currently using no kinds of antibiotics or immunosuppressive medications were included into the study. All patients diagnosed to have cancer or those receiving antibiotics or immunosuppressive therapy during the study period were excluded from the study.

The collected information included patients' demographics and the underlying disease including diabetes mellitus (DM), hypertension, lupus erythematosus, Chronic tubulointerstitial nephritis (TIN), focal segmental glomerulosclerosis (FSGS), polycystic kidneys, glomerulonephritis, and renal stone with and without UTI. Moreover, the type of hemodialysis access was recorded in all patients.

\section{Laboratory methods}

Swabs were taken from the anterior nares of the nose by the Medical Laboratory Technologist. The nasal culture was performed at a recent referring the hospital for hemodialysis (immediately after hemodialysis procedure). After transfer to the laboratory, swabs were placed on blood agar base plates (Biolife Italiana, Milano, Italy) supplemented with $5 \%$ defibriated sheep blood (Darvash Co., Tehran, Iran) and incubated at $35^{\circ} \mathrm{C} \pm 2$ for 48 hours. Suspected colonies were identificated as Staphylococcus aureus by colony morphology, catalase production, and DNase test. $S$. aureus isolates were screened for methicillin resistance (MRSA) following the Clinical Laboratory Standards Institute (CLSI) disk-diffusion method. Overnight cultures from sheep blood agar plates were plated on Mueller-Hinton agar and a $30 \mu \mathrm{g}$ cefoxitin disk (surrogate test for oxacillin) was placed on the inoculated plate. Zone diameters were measured and recorded after 24 hour incubation at $35^{\circ} \mathrm{C} \pm 2$ as susceptible (greater than $22 \mathrm{~mm}$ ) or resistant (less than $21 \mathrm{~mm}$ ). 
Sampling was performed by one trained nurse for all participants by the same protocol. The sampling was performed at the two time points as baseline as well as after a three-month follow up time.

After the results of nasal fluid culture were proven, patients were divided into two groups of negative and positive culture results, and were both followed for 3 months regarding BSI. The results of nasal fluid cultures were then compared with the etiology of BSI in follow-up.

\section{Statistical analysis}

The collected data were reported by mean \pm standard deviation (SD), and percentage. Kolmogorov-Smirnov test was used to assess the normal distribution of data, which showed normal distribution of all data. Thus, continuous variables were compared using $\mathrm{T}$ test and categorical variables using chi-square test. The association of variables were tested by odds ratio and $95 \%$ confidence interval (CI). For the statistical analysis, the statistical software IBM SPSS Statistics for Windows, Version 21.0. Armonk, NY: IBM Corp. was used. P values less than 0.05 were considered statistically significant.

\section{Results}

A total of 63 patients entered analysis. The mean age of participants as $62.79 \pm 16.18$ (range: $24-86)$ years; 32 cases (43.8\%) were female and 31(42.5\%) were male. The most common underlying disease was diabetes mellitus in 22 cases (34.9\%) (Table 1).

Mean duration of hemodialysis was 54.89 months (median: 32, and range of $10-$ 348 ) months. Hemodialysis access route was AVF or AVG in 44 patients (69.8\%), and catheter in 19 patients (30.2\%).

The results of first nasal fluid sample revealed that 21 patients $(33.3 \%)$ in first sampling and 17 patients (27.0\%) in second sampling had positive nasal fluid culture. The type of bacteria in all positive cases was Staphylococcus aureus. At 3-month following up, the BSI positivity was revealed to be $4.8 \%$. The rate of BSI in the patients with positive and negative first nasal fluid culture was $9.5 \%$ and $2.4 \%$ respectively indicating no significant association ( $p=0.256)$. The main source for BSI in both groups with and without BSI positivity was CVCs. In total, two out of three cases with positive BSI were infected with methicillin resistant strains. Similarly, we found no significant association between BSI positivity and nasal fluid culture results because of positive BSI revealed in $5.9 \%$ of patients with positive nasal fluid culture and $4.3 \%$ in those with negative nasal fluid culture with no meaningful difference $(\mathrm{p}=0.800)$. In univariate analysis, none of the baseline variables including gender $(p=0.573)$, underlying risk factor $(p=0.555)$, access $(p=0.214)$, age $(p=0.353)$, or duration of dialysis $(\mathrm{p}=0.682)$ was associated with BSI positivity (Table 2$)$.

Figure 1 shows the frequency of bloodstream infection in the patients with nasal culture in two steps.

\begin{tabular}{|c|c|}
\hline Table 1: Baseline characteristics of study subjects $(\mathrm{n}=63)$. \\
\hline Male gender & $31(49.2)$ \\
\hline Mean age, year & $62.79 \pm 16.19$ \\
\hline Mean duration of dialysis (months) & $45.89 \pm 10.48$ \\
\hline Route of access & $44(69.8)$ \\
\hline AVF or AVG & $19(30.2)$ \\
\hline Catheter & \\
\hline Causes of ESRD & $22(34.9)$ \\
\hline Diabetes mellitus & $6(9.5)$ \\
\hline Glomerulonephritis & $5(7.9)$ \\
\hline Poly kidney disease & $2(3.2)$ \\
\hline Renal stone & $1(1.6)$ \\
\hline Hypertension & $1(1.6)$ \\
\hline ATN & \\
\hline
\end{tabular}




\begin{tabular}{|c|c|c|c|}
\hline \multicolumn{5}{|c|}{ Table 2: Comparing baseline variables between the groups with and without BSI $(\mathbf{n}=63)}$. \\
\hline Item & $\begin{array}{c}\text { BSI }(+) \\
(\mathbf{n}=3)\end{array}$ & $\begin{array}{c}\text { BSI }(-) \\
(\mathbf{n}=60)\end{array}$ & P value \\
\hline Male gender & $1(33.3)$ & $30(50.0)$ & 0.998 \\
\hline Mean age, year & $71.33 \pm 11.85$ & $62.37 \pm 16.33$ & 0.319 \\
\hline Mean duration of dialysis (months) & $39.00 \pm 24.64$ & $55.69 \pm 69.42$ & 0.379 \\
\hline Route of access & & & 0.214 \\
\hline AVF or AVG & $1(33.3)$ & $43(71.7)$ & \\
\hline Catheter & $2(66.7)$ & $17(28.3)$ & \\
\hline History of diabetes mellitus & $3(100)$ & $19(31.7)$ & 0.176 \\
\hline
\end{tabular}

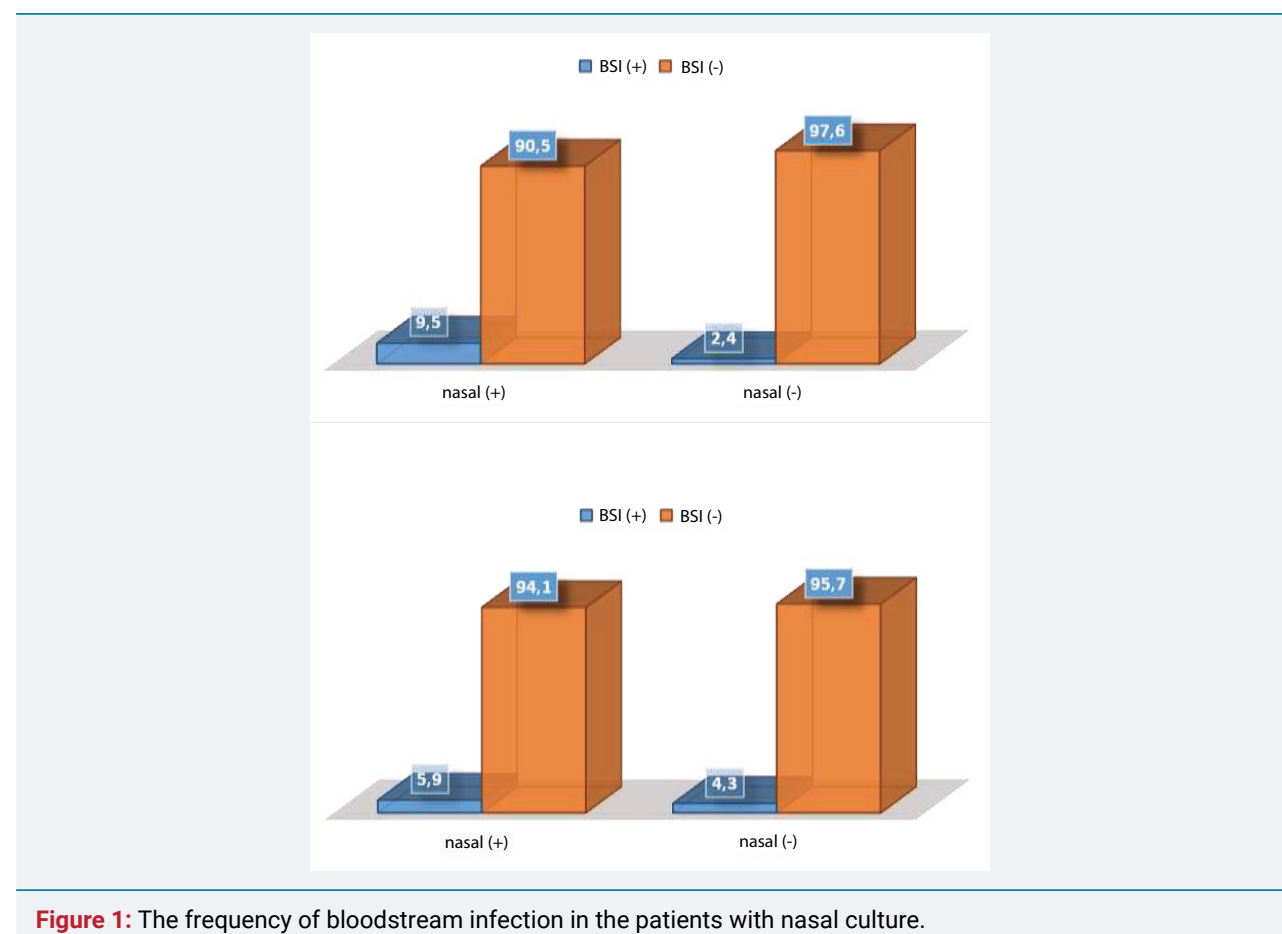

\section{Discussion}

As the main point in our study, we firstly suggested that the presence of positive nasal fluid culture in hemodialysis patients could predict which patients are prone to future positive blood stream infection as an important adverse events of dialysis in such patients, but our suggestion failed and therefore, blood stream infection could not be predicted by nasal fluid culture positivity, at least within three months after primary evaluation. However, some earlier studies had demonstrated our suggestion. As previously pointed, Pujol et al. [15], could show that nasal carriers of S. aureus are at increased risk for bacteremia. One of the main causes for this contradictory results may be sourced from our small sample sizes and thus presence of a low numbers of patients with positive BSI. Another issue may refer to the differences in applying different techniques for detection of bacterimia or to the difference in exclusion or eligibity criteria for inclusion of patients into the study.

The estimated prevalence of BSI in hemodialysis patients varies from country to country, and even from one hospital to another, but the reports of other studies, especially in developing countries indicate higher rates of BSI than the present study. In the United States, patients on hemodialysis had higher rates of bacteremia than peritoneal dialysis and $50 \%$ of patients experienced at least one episode of BSI [17]. Fram and colleagues reported an incidence of $42 \%$ for BSI in hemodialysis patients during six months (10) and other researchers reported the BSI rate at 0.52 per 1000 patient-days [18], which is much higher than the incidence of BSI in our study, which can reflect the appropriateness of preventive health measures taken in Pars hospital's 
dialysis center, including adequate governance structures, multidisciplinary team, and access to expert advice to consider CDC recommendation. Nevertheless, a multicentric study in Iran revealed serious negligence of health guidelines in hemodialysis centers [19], which confirms that the health care provided for hemodialysis patients severely affects the incidence of BSI in these patients and requires great attention.

Also, there were no cases of mortality during the three-month' follow-up in the present study, while other studies have reported the mortality rate ranging from $12-25 \%[20,21]$. The low rate of BSI and no cases of mortality in the present study could also be attributed to the use of AVF as hemodialysis access route (71\%), which is suggested to decrease infection rate, and mortality, compared to CVCs and AVGs [6,7].

In present study, the results of the first nasal culture revealed positive culture in $33 \%$ of subjects, and in the second culture in nearly $27 \%$ of subjects. The discrepancy in the reported rate of positive nasal canal culture in the present study can be explained by the fact that only $20 \%$ of the patients who are carriers of $S$. aureus are persistent carriers, $30 \%$ are intermittent carriers, and 50\% are non-carriers [22]. Alcelik et al., investigated the nasal and external ear canal cultures of 83 hemodialysis patients with mean age of 61 years and reported positive nasal canal culture (coagulase- negative staphylococci and diphteroids) in $25 \%$ of participants, with $81.3 \%$ similar microflora in the nasal and external auditory canal [14]. The mean age of participants of their study was similar to ours and their percentage on positive nasal canal culture was similar to our results, in the second nasal culture. Verhoeven and colleagues reported S. aureus in 18/76 hemodialysis patients (23.6\%) during a one year follow-up, which is also close to the second nasal canal culture in the present study. They also proposed an algorithm for taking nasal fluid culture once a week during a 10-week period based on quantitative polymerase chain reaction with a sensitivity, and specifity of $95.8 \%$, and $94.2 \%$, respectively [12]. Therefore, it is necessary to pay great attention to nasal fluid specimens in hemodialysis patients. A meta-analysis on six published cohort studies indicated that among 1150 hemodialysis patients, methicillin-resistant S. aureus (MRSA) was isolated from nasal swabs of 147 (12.8\%) patients [23], which is lower than the percentages in the present study. This difference is due to the fact that we have reported the whole infections and they have considered MRSA as the most important pathogen in resistant BSI.

In present study, in all positive cases of the first nasal culture and nearly $95 \%$ of the second culture, the type of infection was $S$. aureus, which is similar to previous studies indicating as prevalent causes of bacteremia to be Gram positive micro-organism, responsible for 96 episodes (65\%), with $S$. aureus as the most frequent (55\%), followed by S. epidermidis (26\%) and Gram-negative for 36 (23\%), with E. coli (39\%) as the most frequent agent, while $9.5 \%$ were polymicrobial infection [16]. Therefore, it can be concluded that Gram positive micro-organisms, especially $S$. aureus the most important organism, which call for adequate preventive and treatment measures.

Although the present prospective study addressed an important issue and prediction of future BSI by nasal fluid culture can help decrease the rate of mortality and morbidity of hemodialysis patients, the present study had several limitations, including small sample size. In addition, the study recruited patients from one center that could clearly show the prophylactic policy of that center, but it could limit the generalizability of the results. Therefore, it is suggested that future multicentric studies address this issue with larger sample size, and longer follow-up. Due to this fact that small sample size is a potential limitation of the study because of no more patients available, implementing further studies with larger sizes is recommended. Also, due to the considerable difference in the sensitivity of culture and the PCR technique in detection of bacterial strains, applying both techniques especially the PCR to identify bacterial genome is recommended in future studies. 


\section{Conclusion}

In conclusion, the results of the present study indicated BSI in 3 out of 63 hemodialysis patients in a three-month' follow-up, while nasal canal culture was not significantly associated with it, which was possibly due to the small number of BSI cases. According to our result, BSI may not be predicted by nasal fluid culture positivity particularly in hemodialysis patients.

\section{Acknowledgements}

The authors of the present study sincerely thank the SMART Company for their technical support for implementing the plan and other colleagues who helped us in the study process.

Disclosure of grants or other funding: The present study was financially supported by SMART Company.

\section{References}

1. Abdel-Kader K, Unruh ML, Weisbord SD. Symptom burden, depression, and quality of life in chronic and end-stage kidney disease. Clinical Journal of the American Society of Nephrology. 2009; 4: 1057-1064. Ref.: http://tinyurl.com/y6oxqmhz

2. Wasse H. Catheter-Related Mortality among ESRD Patients. Semin Dial. 2008; Wiley Online Library. 21: 547-549. Ref.: http://tinyurl.com/yx9g73we

3. Dalrymple LS, Mu Y, Romano PS, Nguyen DV, Chertow GM, et al. Outcomes of infection-related hospitalization in Medicare beneficiaries receiving in-center hemodialysis. Am J Kidney Dis. 2015; 65: 754-762. Ref.: http://tinyurl.com/y23173uf

4. Leone S, Suter F. Severe bacterial infections in haemodialysis patients. Infez Med. 2010; 18: 79-85 Ref.: http://tinyurl.com/yxfev8nm

5. Karkar A. Infection control guidelines in hemodialysis facilities. Kidney Res Clin Pract. 2018; 37: 1-3. Ref.: http://tinyurl.com/yxlnrvzp

6. Patel PR, Brinsley-Rainisch K. The Making Dialysis Safer for Patients Coalition: A New Partnership to Prevent Hemodialysis-Related Infections. Clin J Am Soc Nephrol. 2017; pii: CJN.02730317. Ref.: http://tinyurl.com/yyuxch6f

7. Nguyen DB, Gutowski J, Ghiselli M, Cheng T, Bel Hamdounia S, et al. A Large Outbreak of Hepatitis C Virus Infections in a Hemodialysis Clinic. Infect Control Hosp Epidemiol. 2016; 37: 125-133. Ref.: http://tinyurl.com/y6fncgoh

8. Malyszko J, Zawierucha J, Marcinkowski W, Prystacki T, Dryl-Rydzynska T, et al. The prevalence of hepatitis $\mathrm{C}$ and $\mathrm{B}$ among patients on hemodialysis and on renal transplantation waiting list in Poland has significantly decreased during the last 10 years. Int Urol Nephrol. 2018; 50: 1555-1556. Ref.: http://tinyurl.com/y2wjxtla

9. Dalrymple LS, Mu Y, Nguyen DV, Romano PS, Chertow GM, et al. Risk Factors for Infection-Related Hospitalization in In-Center Hemodialysis. Clinical journal of the American Society of Nephrology: CJASN. 2015; 10: 2170-2180. Ref.: http://tinyurl.com/y2d5stoc

10. Fram D, Taminato M, Ponzio V, Manfredi SR, Grothe C, et al. Risk factors for morbidity and mortality of bloodstream infection in patients undergoing hemodialysis: a nested case-control study. BMC research notes. 2014; 7: 882. Ref.: http://tinyurl.com/y3pukkrk

11. Schmid H, Romanos A, Schiffl H, Lederer SR. Persistent nasal methicillin-resistant staphylococcus aureus carriage in hemodialysis outpatients: a predictor of worse outcome. BMC nephrology. 2013 14: 93. Ref.: http://tinyurl.com/y45rj74f

12. Verhoeven PO, Gagnaire J, Haddar $\mathrm{CH}$, Grattard F, Thibaudin D, et al. Identifying Hemodialysis Patients With the Highest Risk of Staphylococcus aureus Endogenous Infection Through a Simple Nasal Sampling Algorithm. Medicine. 2016; 95: e3231. Ref.: http://tinyurl.com/y2w3awhh

13. Grothe C, Taminato M, Belasco A, Sesso R, Barbosa D. Screening and treatment for Staphylococcus aureus in patients undergoing hemodialysis: a systematic review and meta-analysis. BMC nephrology. 2014; 15: 202. Ref.: http://tinyurl.com/y2wqakda

14. Alcelik A, Apuhan T, Kocoglu E, Eroglu M, Necati Hakyemez I, et al. Microbial colonization of the external auditory canal and nose in hemodialysis patients. Medicinski glasnik: official publication of 
the Medical Association of Zenica-Doboj Canton, Bosnia and Herzegovina. 2012; 9: 330-333. Ref.: http://tinyurl.com/yy86p4fm

15. Pujol M, Peña C, Pallares R, Ariza J, Ayats J, et al. Nosocomial Staphylococcus aureus bacteremia among nasal carriers of methicillin-resistant and methicillin-susceptible strains. Am J Med. 1996; 100: 509-516. Ref.: http://tinyurl.com/yyqtuggr

16. Chow JW, Yu VL. Staphylococcus aureus nasal carriage in hemodialysis patients. Its rolein infection and approaches to prophylaxis. Arch Intern Med. 1989; 149: 1258-1262. Ref.: http://tinyurl.com/y6nq7crc

17. Dalrymple LS, Johansen KL, Chertow GM, Cheng SC, Grimes B, et al. Infection-Related Hospitalizations in Older Patients With End-Stage Renal Disease. Am J Kidney Dis. 2010; 56: 522-530. Ref.: http://tinyurl.com/y4mb9b5j

18. Fysaraki M, Samonis G, Valachis A, Daphnis E, Karageorgopoulos DE, et al. Incidence, clinical, microbiological features and outcome of bloodstream infections in patients undergoing hemodialysis. Int J Med Sci. 2013; 10: 1632-1638. Ref.: http://tinyurl.com/yywrqrgg

19. Askarian M, Hajiabadi MJ, Kashkooli YA, Yousofi M, Fathi H, et al. Improving infection prevention methods in hemodialysis units: A multicenter survey. Am J Infect Control. 2014; 42: 193-194. Ref.: http://tinyurl.com/y29t5bm3

20. Maki DG, Kluger DM, Crnich CJ. The risk of bloodstream infection in adults with different intravascular devices: a systematic review of 200 published prospective studies. Mayo Clin Proc. 2006; 81: 11591171. Ref.: http://tinyurl.com/y53d8j9o

21. Gahlot R, Nigam C, Kumar V, Yadav G, Anupurba S. Catheter-related bloodstream infections. Int J Crit Illn Inj Sci. 2014; 4: 162-167. Ref.: http://tinyurl.com/y28k5ct5

22. Wertheim HF, Melles DC, Vos MC, van Leeuwen W, van Belkum A, et al. The role of nasal carriage in Staphylococcus aureus infections. Lancet Infect Dis. 2005; 5: 751-762. Ref.: http://tinyurl.com/y33ergtl

23. Gebreselassie HM, Lo Priore E, Marschall J. Effectiveness of meticillin-resistant Staphylococcus aureus decolonization in long-term haemodialysis patients: a systematic review and meta-analysis. J Hosp Infect. 2015; 91: 250-256. Ref.: http://tinyurl.com/y5lsbnyo 